International Journal of Surgery and Research (IJSR)

ISSN: 2379-156X

\title{
Omphalomesenteric Duct Remnant As A Cause Of Small Bowel Obstruction With Incidental Appen- diceal Carcinoid: A Case Report
}

Case report

Heuser $\mathrm{J}^{*}$, Lampron J

Department of Surgery, University of Ottawa, Ottawa, ON, Canada

Abstract

Omphalomesenteric duct remnants are a rare cause of abdominal pain, especially in the adult. Their diagnosis is not straightforward. We report the case of a healthy 25 -year-old male presenting with chronic, intermittent abdominal pain over 1 month with no previous abdominal surgeries. Extensive investigations including computed tomography (CT) scan and colonoscopy were non-contributory. Thus a diagnostic laparoscopy was performed, which revealed a fibrous band originating from the anterior abdominal wall at the umbilicus and terminating behind the mid-transverse colon, with cecum and small bowel tethered to it, as well as signs of chronic inflammation in this area. Excision of the band as well as an appendectomy were performed. Histopathology confirmed the specimen to be an omphalomesenteric (vitelline) duct remnant, and also revealed an incidental small carcinoid tumour in the appendix. This case report highlights that undiagnosed abdominal pain with negative investigations needs to be treated with caution to not miss a broader range of diagnoses and that diagnostic laparoscopy is a useful tool. Omphalomesenteric duct remnant is a rare cause of chronic abdominal pain and intermittent obstruction in an adult.

Key Words: Omphalomesenteric Duct Remnant, Intestinal Obstruction, Diagnostic Laparoscopy, Carcinoid Tumour

\section{*Corresponding Author:}

Jordan Heuser,

Department of Surgery,

University of Ottawa, Ottawa, ON, Canada.

E-mail: jordan.heuser@gmail.com

Received: August 28, 2014

Accepted: September 19, 2014

Published: September 20,2014

Citation: Heuser J, Lampron J. (2014). Omphalomesenteric Duct Remnant As A Cause Of Small Bowel Obstruction With Incidental Appendiceal Carcinoid: A Case Report, Int J Surg Res, 01(01), 01-04. doi: http: / / dx.doi.org/10.19070/2379-156X-140001

Copyright: Heuser $\mathbf{J}^{\odot}$ 2014. This is an open-access article distributed under the terms of the Creative Commons Attribution License, which permits unrestricted use, distribution and reproduction in any medium, provided the original author and source are credited.

\section{Background}

This is an important and educational case for several reasons. Omphalomesenteric duct remnants are a rare congenital anomaly and even more rarely are a cause of abdominal pain and small bowel obstruction in adults. Omphalomesenteric duct remnants have a wide range of presentations, from asymptomatic to Meckel's diverticulum or enterocutaneous fistula. The utility of diagnostic laparoscopy in this setting is emphasized. Furthermore, this case report emphasizes the management of appendiceal carcinoid tumours, which are the most frequently diagnosed tumour of the appendix (25-50\%), although still rare and found incidentally in only $0.3-0.9 \%$ of appendectomies. [1-4] To our knowledge, this is the first case reported in the literature of a simultaneous omphalomesenteric duct remnant and appendiceal carcinoid tumour in the context of an adult with abdominal pain.

\section{Case Presentation}

The patient is a 25-year-old male previously well who presents to the Emergency Department (ED) for the fourth time within the last month with complaints of non-specific, generalized, intermittent abdominal pain over this period associated with occasional nausea, vomiting and anorexia. His weight was stable and he denied any diarrhea. Investigations included normal laboratory tests, ultrasound with no sign of acute appendicitis and a CT scan. The CT scan showed a small to moderate amount of pelvic free fluid and also commented on difficulty following the large bowel with a relatively midline cecum. A colonoscopy was also performed that was unremarkable except for redundant colon with possible twisting of the colon at 70 centimetres and dilation proximal to this area of twisting. Subsequent review of the patient's CT scan prompted the patient to be called back to the ED for further investigations, including paracentesis of the abdominal free fluid. The gram stain from the paracentesis showed only neutrophils, cultures demonstrated no growth and cytologic examination was negative for malignancy. During this time he was well, pain-free and tolerating a full diet. However, he returned to the hospital one week later with a similar pattern of symptoms. His laboratory values included white blood cells of $7.7 \times 10^{9} / \mathrm{L}$ and normal bilirubin, transaminases and lipase. Abdominal series revealed no dilated small or large bowel, no air-fluid levels, and no other signs of obstruction. Given the recurrent pattern of his pain, unremarkable investigations and equivocal CT and colonoscopies findings in an otherwise healthy male with no previous abdominal surgeries, the option of diagnostic laparoscopy was offered to the patient.

\section{Treatment}

The laparoscopy revealed a fixed adhesive fibrous band from the right lower quadrant passing over the terminal ileum and ceacum, causing partial obstruction as well as twisting of the appendix around this band. The band continued behind the transverse co- 
lon. Upon applying traction to identify the insertion of the fibrous band, it broke loose at the proximal end. The band followed the white line of Toldt along the ascending colon and then turned to end behind the transverse colon. We released this attachment and removed the fibrous band measuring approximately $40 \mathrm{~cm}$. Intraoperative frozen section of this band was negative for any vascular or mucosal structures. Even though we had a satisfying cause to explain the symptoms of the patient, a decision was made to perform an appendectomy.

\section{Outcome}

Final pathology revealed that the band was an omphalomesenteric (vitelline) duct remnant, and surprisingly and unexpectedly we discovered a small, $2 \mathrm{~mm}$ neuroendocrine tumour, likely carcinoid, in the mid appendix. The margins of the appendix were negative. The patient did well post-operatively with resolution of his intermittent obstructive symptoms. He was still well after five weeks and given the small nature of the carcinoid tumour that had been completely resected, no further treatment or follow-up was recommended.

\section{Timeline}

\begin{tabular}{|l|l|}
\hline First presentation to ER & Day 1 \\
\hline US & \\
\hline Second visit to ER & Day 11 \\
\hline CT scan & \\
\hline Colonoscopy & Day 12 \\
\hline Third visit to ER & Day 13 \\
\hline Paracentesis & Day 14 \\
\hline Fourth visit to ER, admitted & Day 19 \\
\hline Diagnostic laparoscopy & \\
\hline Discharged home & Day 20 \\
\hline Follow-up & Day 62 \\
\hline
\end{tabular}

\section{Discussion}

This case report highlights several less commonly encountered topics in adult surgical patients. Firstly, the management of obstructive symptoms in a patient with a virgin abdomen is reinforced. The differential diagnosis of course includes neoplasm,

Figure 1. Intra-operative pictures. (A) Band partially obstructing the terminal ileum and caecum. (B) Long fibrous cord after being released

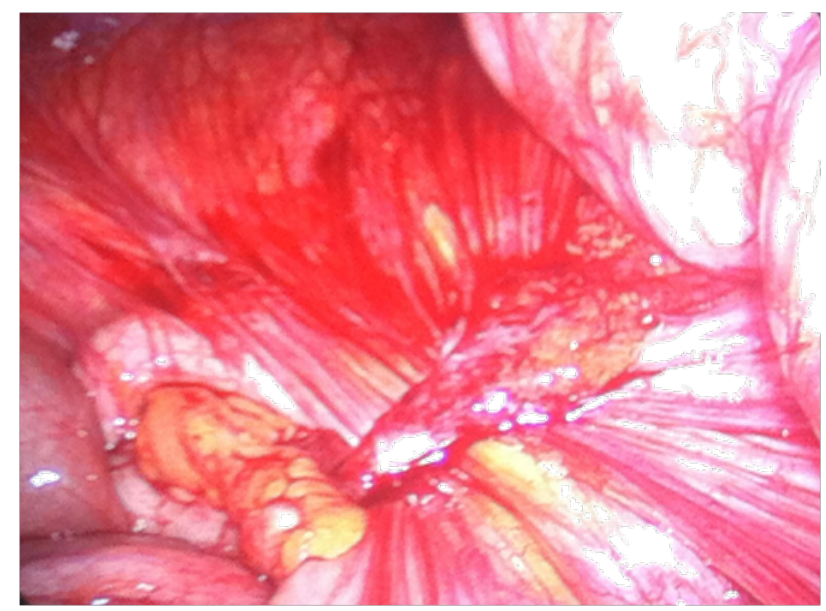

A

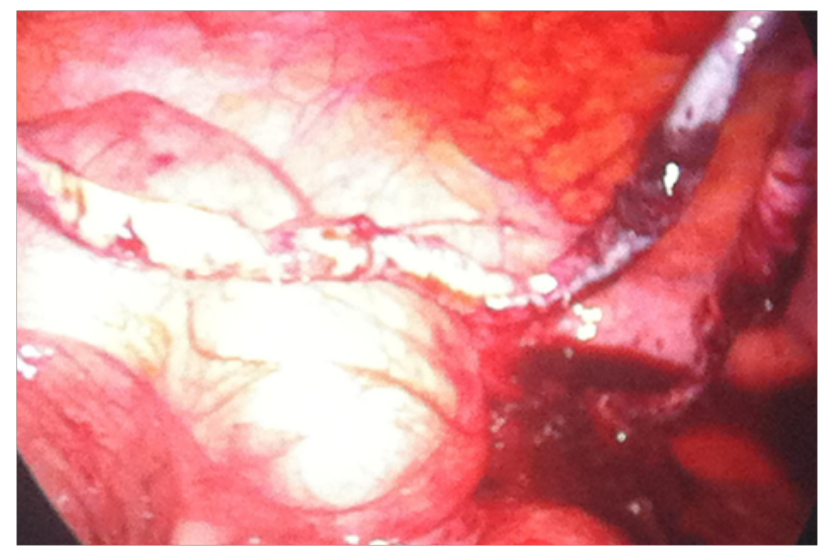

B

hernia, post-inflammatory adhesions, and Crohn's disease. A recent retrospective analysis by Beardlsey and colleagues of all causes of small bowel obstruction in patients without previous abdominal surgery revealed post-inflammatory adhesions to be the most common cause $(76 \%)$ versus a newly diagnosed malignancy as the second most common cause $(10 \%)$, excluding incarcerated external hernias from their analysis.[5] Less common etiologies include bezoar, foreign body, gallstone ileus, and intussusception. We suggest another potential cause that should be considered in such a diagnostic challenge is a congenital band, such as a remnant omphalomesenteric duct.

Congenital anomalies remain a rare cause of pathology in adults, but among the most common are omphalomesenteric duct anomalies. There are a variety of omphalomesenteric duct anomalies, as depicted in Figure 2, including fistula, Meckel's diverticulum and a fibrous cord.[6] 
Figure 2. Varieties of omphalomesenteric duct remnant malformations.[6] Reproduced with permission from Elsevier
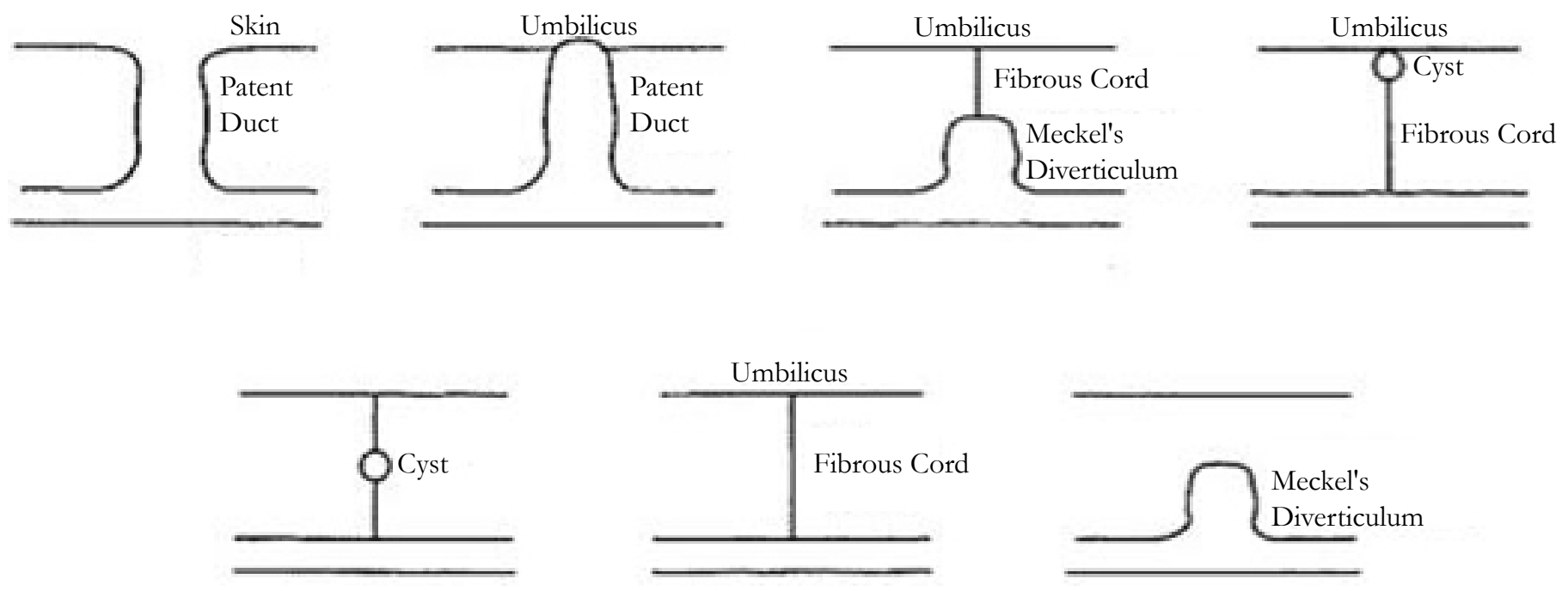

Of note, the omphalomesenteric tract also includes an artery and vein. These vessels are obliterated in the majority of cases; however, caution must be taken when ligating such a structure to ensure proper hemostasis.[7] Pathology secondary to a remnant omphalomesenteric tract is more common in children and becomes increasingly less common with age. [6] It is unusual that our patient's symptoms began only one month prior to presentation, thus necessitating a high index of suspicion.

In our case, advanced diagnostic procedures including abdominal ultrasound, CT scan, paracentesis and colonoscopy were not conclusive in making a diagnosis.

As outlined by Beardlsey and colleagues in their analysis, laparotomy is often considered mandatory for patients with small bowel obstruction and no previous abdominal surgeries.[5] However, they argue for a trial of non-operative or conservative management in select patients since post-inflammatory adhesions are the most common cause. We would suggest the addition of diagnostic laparotomy to the diagnostic armamentarium in such patients.

An appendectomy was performed to eliminate appendicitis as a differential diagnosis should the patient experience any further episodes of abdominal pain.

Naturally, this was the first diagnosis considered when the patient presented originally. Furthermore, the scars remaining from the lysis of adhesions and resection of the congenital band may have led to confusion in the future since the scar pattern would resemble that of a laparoscopic appendectomy.

The carcinoid tumour found unexpectedly on pathology highlights another important surgical topic. Carcinoid tumours are the most common tumour of the appendix, comprising approximately $25-50 \%$ of all appendiceal tumours. The appendix is also the most common location for carcinoid tumours to be found, with the second most common location being the ileum. Overall however, appendiceal carcinoid remains an uncommon finding in only $0.3-0.9 \%$ of specimens. The majority are found in the distal third of the appendix, arising from subepithelial neuroendocrine cells. They also usually exhibit an indolent course, and thus are primarily found incidentally upon appendectomy or resection for other reasons. [1,3] Unfavourable features on histopathological review include size greater than $2 \mathrm{~cm}$, evidence of mesoappendiceal extension, tumours near the appendiceal base with positive margins or caecal involvement, high mitotic indices, and evidence of lymphovascular invasion. [3,8] If any of these higher risk features are present, consideration of ileocecectomy or right hemicolectomy is recommended. However, if none of these features are evident as in our case, then simple appendectomy is sufficient. $[3,4,9]$

To our knowledge, there is no other case reporting omphalomesenteric duct pathology associated with an appendiceal carcinoid tumour.

In order of quality and rigor, this case report follows the principles of the CARE statement.

\section{Learning Points}

- Omphalomesenteric duct remnant must be considered as an uncommon but potentially serious cause of abdominal pain in adult.

- Diagnostic laparoscopy is a good diagnostic tool even if other tests are non contributory

- Prophylactic appendectomy in diagnostic laparoscopy might be beneficial

\section{Conclusion}

In summary, we believe that diagnostic laparoscopy is an important diagnostic tool in the case of unexplained abdominal pain in an adult with no previous abdominal surgeries. In addition, this case upholds the benefit of performing an appendectomy even when there is another clear explanation of the patient's symptoms.

\section{Patient Consent}

Written informed consent was obtained from the patient for publication of this case report and any accompanying images.

\section{References}

[1]. Modlin IM, Lye KD, Kidd M (2003) A 5-decade analysis of 13,715 carcinoid tumors. Cancer 97: 934-59.

[2]. Connor SJ, Hanna GB, Frizelle FA (1998) Appendiceal tumors: retrospective clinicopathologic analysis of appendiceal tumors from 7,970 appendectomies. Dis Colon Rectum 41: 75-80. 
[3]. Goede AC, Caplin ME, Winslet MC (2003) Carcinoid tumour of the appendix. Br J Surg 90:1317-22.

[4]. In't Hof KH, van der Wal HC, Kazemier G (2008) Carcinoid tumour of the appendix: an analysis of 1485 consecutive emergency appendectomies. J Gastrointest Surg 12:1436-8.

[5]. Beardlsey C, Furtado R, Mosse C (2014) Small bowel obstruction in the virgin abdomen: the need for a mandatory laparotomy explored. Am J Surg 208(2):243-8

[6]. Moore TC (1996) Omphalomesenteric duct malformations. Seminars Ped Surg 5:116-23.
[7]. Jalil O, Radwan R, Rasheed A (2012) Congenital band of the vitelline artery remnant as a cause of chronic lower abdominal pain in an adult: case report. Int J Surg Case Reports 3: 207-8.

[8]. Murray SE, Ricardo VL, Sippel RS (2013) Postoperative surveillance of small appendiceal carcinoid tumors. Am J Surg 207(3):342-5

[9]. Kulke MH, Mayer RJ (1999) Carcinoid tumors. N Engl J Med 340: 858-68. 